\title{
Perimembranous ventricular septal defect closure via ultra-minimal trans intercostal incision in children
}

\author{
Zhan $\mathrm{Gao}^{1}$, Jin $\mathrm{Yu}^{1}$, Zewei Zhang ${ }^{1}$, Jianhua $\mathrm{Li}^{1}$, and Jiangen $\mathrm{Yu}^{1}$ \\ ${ }^{1}$ Affiliation not available
}

June 6, 2021

\begin{abstract}
Aims:This study aimed to investigate the safety, feasibility and availability of perimembranous ventricular septal defect (PmVSD) closure via a left parasternal ultra-minimal trans intercostal incision in children. Methods and results:From January 2015 to January 2019, 131 children with restrictive PmVSDs were enrolled in this study and successfully done in 126 patients (96.18\%). PmVSDs were occluded via an ultra-minimal trans intercostal incision ([?]1 cm), and the entire occlusive process was guided and monitored by TEE. A pericardium hanging technique was employed without sternal incision. PmVSDs were closed through a short delivery sheath assembled using a concentric occluder device. All patients were followed up for a perid ranging from18 months to 24 months. Thirteen patients with PmVSD had aneurysm of membranous septum (AMS). Multistream (more than or equal to 2) PmVSDs with AMS were found in eleven cases. After the operation, mild residual shunt beside the amplatzer occluder in one patient was found and had self-healing result during the 5 -month follow-up period. Five patients transferred to ventricular septal defect repair operation under direct visualization with a cardiopulmonary bypass. One reason was ventricular fibrillation when guide wire passed the PmVSD, another was device dislocation, and others were the guide wire cannot pass through the PmVSD. Conclusions:PmVSDs closure using a concentric occluder via a left parasternal ultra-minimal trans intercostal incision under TEE guidance is feasible, safe, and effective in children. This approach can be considered as an alternative treatment to open-heart surgery for restrictive PmVSDs.
\end{abstract}

\section{Introduction}

Ventricular septal defect (VSD) is a common congenital heart disease in children, and most VSDs are perimembranous ventricular septal defects (PmVSDs).

Traditionally, PmVSDs are surgically repaired under direct visualization with cardiopulmonary bypass. Although this surgery is effective, it is associated with significant trauma and slow postoperative recovery.Blood transfusion is necessary in the cardiopulmonary bypass approach.

Alternatively, the percutaneous closure of a PmVSD has the advantages of no blood transfusion, minimal trauma and rapid recovery ${ }^{[1,2]}$. But it is associated with exposure of radiation and allergy to contrast agent $^{[3,4]}$. Another disadvantage of the percutaneous closure technique is that it is a complicated approach requiring an arteriovenous guidewire loop; this procedure cannot be performed in patients with vascular access problems.

In recent years, PmVSDs closure via transthoracic minimally invasive incision under transesophageal echocardiography(TEE) guidance has attracted the attention of many scholars ${ }^{[5-10]}$. This approach does not require cardiopulmonary bypass and radiation; it has the advantages of a short delivery path and no restriction on weight or vascular access. It is particularly advantageous for children patients who cannot be treated with percutaneous catheter closure or patients who cannot tolerate surgery and cardiopulmonary bypass. However, this surgical method requires the sternum to be transected, which easily causes postoperative bleeding, 
pain, and pectus excavatum ${ }^{[11]}$. Moreover, the incision length can reach approximately $2-3 \mathrm{~cm}$, which affects appearance. Therefore, the procedure cannot be considered as ideal in terms of minimally invasive surgery.

Based on the minimally invasive transthoracic closures of nearly 1000 patients, our team modified this surgical incision as follows: a less than $1 \mathrm{~cm}$ trans intercostal incision in the left sternal margin. Via an ultra-minimal trans intercostal incision and a pericardium hanging technique, PmVSDs had been successfully occluded using the concentric occluder devices under TEE guidance. In this study, we aimed to investigate the safety, feasibility and availability of this new surgical approach in children.

\section{Material and methods}

\section{Patient inclusion}

A total of 131 children who underwent PmVSD closure via a left parasternal ultra-minimal intercostal incision between January 2015 to January 2019 were included in this study. The children patients were diagnosed with restrictive PmVSD based on clinical signs, transthoracic echocardiography (TTE), electrocardiograms and X-rays.

The inclusion criteria were as follows: no obvious reduction of the size of PmVSD,slow weight gain and loud heart murmurs; no or mild tricuspid regurgitation(TR) without valvular heart disease; the distance between the PmVSD upper rim and the aortic valve larger than $2 \mathrm{~mm}$. The exclusion criteria were as follows: nonrestrictive VSD; malalignment-type VSD; valve abnormality that require surgical repair; severe pulmonary hypertension and other heart diseases; other surgical contraindications. Informed consent was obtained from all the guardians of the patients.

\section{Surgical procedures}

\section{Device selection}

Final operation plan of PmVSD was chosen according to the TEE results of the patient after anesthesia in the operating room (Fig. 3A). The size, location, morphology, and surrounding tissue of PmVSD were comprehensively evaluated. For PmVSD with AMS, the diameter, number and direction of the defect in the AMS and the distance between the defects were seriously analyzed. Simultaneously, the left ventricular side diameter of AMS was carefully measured. A proper concentric occluder device size was chosen according to the diameter of the VSD by TEE measure. Usually, the waist sizes of devices were 0.5-1.5 mm larger than the VSD maximum diameters. For the patients who had multistream (more than or equal to 2) PmVSDs in the AMS, the occluder was selected according to the specific circumstances such as the multistream maximum distance and the left ventricular side diameter of AMS.

\section{Device and delivery system}

The closure device and delivery system used in this study was a domestic concentric occluder (Shanghai Shape Memory Alloy Co., Ltd., Shanghai, China, and Shanghai Push Medical Device Technology Co., Ltd., Shanghai, China). The minimum size of the occluder was $4 \mathrm{~mm}$. Both disks of the concentric occluder have a diameter of $2 \mathrm{~mm}$ larger than that of the waist (Fig. 1). The waist is 3.5-4.5 mm in height. The entire delivery system consisted of a trocar, a guidewire, a delivery cable, a short loading sheath, a long delivery sheath and a dilator.

Before using, the selected concentric occluder must be immersed in heparin saline, and the delivery system must be washed with heparin saline. The selected occluder was screwed onto the delivery cable which had been inserted into the loading sheath. Then, the procedure of smooth release and withdrawn of the device must be examined prior to the surgery via the device being pushed forward out the sheath and pulled back into the sheath. In order to improve the safety of this closure technique, a device protective suture of 3-0 polypropylene was passed through the mesh of the right disk near the middle of every device. It has to be emphasized that the suture cannot entwine the delivery cable as it was pulled out of the sheath.

\section{Procedure}


The patient's body was tilted to the left, and the right side of the back was slightly elevated. TTE was used to determine the position of the trans intercostal incision based on the location of VSD and the main direction of blood flow. A surgical incision [?] $1 \mathrm{~cm}$ in length (the minimum incision in this study was $0.7 \mathrm{~cm}$ ) was made at the left margin of sternum of the $3 \mathrm{rd}$ to the 5 th intercostal spaces, normally the 4 th intercostal space (Fig.2B, Fig.2C).

The subcutaneous soft tissues were opened by blunt dissection without entering into the pleural cavity. The pericardium was transversely incised and hung with 4-5 sutures (Fig.2A). TEE was used to determine the position of the purse-string suture on the right ventricular surface. With the real-time guidance of TEE, the cardiac surgeon moved the tweezers in order for the tweezers' head to point toward the PmVSD and the main direction of blood flow. An optimal angle from the selected site toward the VSD facilitated the guidewire passing through the VSD.

After heparin $(1 \mathrm{mg} / \mathrm{kg})$ was administered, a purse-string suture was made at the position of the tweezers' head under direct visualization. TEE was placed at a suitable angle for real-time monitoring and guidance. A punch needle was inserted into the right ventricle through the pouch. Then, the guidewire was sent to the left ventricle through the PmVSD (Fig. 3b). The delivery sheath and the dilator were introduced to the left ventricle over the guidewire after the puncture needle was withdrawn. If the defect diameter of VSD was too small, surgeon should expand VSD using the bigger dilator so that the delivery sheath pass through the PmVSD smoothly. While the top of the sheath was confirmed to be in the left ventricle, the guidewire and the dilator were withdrawn (Fig. 3C. The short loading sheath was then connected to the long delivery sheath. The occluder was pushed to released the left disk from the sheath (Fig. 3D). According to the shape of PmVSD and AMS, the left disk was placed on the left ventricular side of PmVSD or pulled all or partly into the aneurysm. Then, the delivery sheath was withdrawn back to the right ventricle, and the waist of the device and the right disk were fully released (Fig. 3E).

TEE was immediately performed. If the device were perfectly released without complications such as device dislocation, residual shunt, device-related valve regurgitation (especially the tricuspid valve) and arrhythmia, the device was inspected repeatedly by a push-pull maneuver and unscrewed from the delivery cable. Otherwise, the occluder was adjusted, withdrawn, or replaced if complications arose.

After the occluder was released and unscrewed from the delivery cable, the protective device suture contributed to the retrieval of the device through a larger delivery sheath if the device was found to be displaced by TEE. If all went well, the suture was gently pulled out from the device and the delivery device was withdrawn. The last steps were to ligate the purse-string suture and close the incision in layers (Fig. 3).

ECG monitor was used to monitor heart rhythm and blood pressure during the operation. During the surgery, care should be taken in monitoring heart rhythm, blood pressure, oxygen saturation, blood gas analysis and airway management. If these indications are abnormal, the procedure should be discontinued and should even be cancelled.

\section{Follow-up}

All patients were followed up. TTE, electrocardiograms, X-rays and blood tests were used to assess surgical outcomes. The follow-up exams assessed heart function, heart rhythm, occluder placement, residual shunt, valve regurgitation, infective endocarditis, thrombus and pericardial effusion.

\section{Results}

126 patients were successfully operated by an ultra-minimal trans intercostal device closure in all 131 cases. Operation success rate is $96.18 \%$.

13 patients with PmVSD had aneurysm of membranous septum (AMS). Single stream PmVSDs, which with or without AMS, were found in 118 cases. Multistream (more than or equal to 2) PmVSDs with AMS were found in eleven cases. All of them were closed using one concentric occluder. There was a mild residual shunt with a diameter of $1.5 \mathrm{~mm}$ and a flowing velocity of $2.0 \mathrm{~m} / \mathrm{s}$ beside the amplatzer occluder after the surgery, 
and it had self healed during the 5-month follow-up period. 9 cases of mild pericardial effusion was found after the operation and all disappeared during the 3-month follow-up period. Three cases had a trasient postoperative hyperpyrexia and returned to normal after treatment. The degree of TR did not aggravate after surgery. The patients with successful surgery had good cardiac function. No blood transfusions, serious arrhythmias or death occurred. Neither did dislocated devices, residual shunt or device-related valve regurgitation.

5 patients transferred to ventricular septal defect repair operation under direct visualization with a cardiopulmonary bypass, and the operations were smooth and the follow-up were normal. One patient was a fat kid of 63-kg-weight and129-month-old. The surgeon pulled on leftwards the heart more than $3 \mathrm{~cm}$ to find the precise position of the purse-string suture. Ventricular fibrillation occured when guide wire passed the PmVSD. Another child patient was a 39-month-old and 15-kg-weight boy with large PmVSD of $7.7 \mathrm{~mm}$ size. The surgeon tried to close the large PmVSD with $8 \mathrm{~mm}$ and $9 \mathrm{~mm}$ large device, but the devices was translocated. The procedure was not performed again using larger device considering his age. Others were the guide wire cannot pass through the PmVSD, so the surgeons can only performed VSD repair under cardiopulmonary bypass.

All patients were followed for a perid ranging from18 months to 24 months. Follow-up examinations showed good placement of the occluder, good heart function, no residual shunts, no arrhythmia, no infective endocarditis and no thrombus. Device-related valve regurgitation were not found.

\section{Discussion}

Our team modified a novel approach of PmVSD closure via ultra-minimal trans intercostal incision. Compared with the minimally invasive inferior sternotomy approach, the operation trauma of novel approach is much less $([?] 1 \mathrm{~cm})$. This novel surgical incision avoids the need for a sternal incision and blood transfusion. The pericardium hanging technique replaced the tissue retractor devices for exposure that can avoid injury to the ribs and the intercostal tissue ${ }^{[12]}$. After the incision healing, it does not affect the appearance and the postoperative mental health of patients. By using ultra-minimal skin incisions and transintercostal incision , surgical trauma can be greatly reduced, Particularly in the pediatric population.

Compared with the transcatheter PmVSD closure approach, the novel approach is simpler. This approach has a short operating pathway of and does not require the establishment of an arteriovenous guidewire loop ${ }^{[12]}$, which can prevent inferior vena cava and aorta injure. It provides an angle point toward the PmVSD, and the guidewire is easier to pass through VSD. Moreover, clear TEE image ${ }^{[12,13]}$ can accurately and effectively assess PmVSD and clearly show the guide wire, sheath and occluder to provide real-time guidance that enables cardiac surgeons to operate smoothly.

The positions of the intercostal incision and the purse-string suture should be selected very carefully and accurately because ultra-minimal incisions ([?]1 cm) limit the range over which the guidewire and sheath can move. Our team used both TTE and TEE to select accurately location of the incision and that of the pursestring suture, according to the location of the PmVSDs and the main direction of blood flow. First, TTE was used to determine the position of the incision. The PmVSDs is often located behind the sternum. So the incision should be located next to the left sternum, simultaneously, the blood vessels and nerve tissues were avoided. Then, TEE was used to determine the purse-string position. Under TEE guidance, the surgeon gently pulled the heart to the left, and found out the position of purse-string suture through tweezer being moved on the right ventricular anterior wall. It should be noted that the surgeons should clamp gently the right ventricular surface using the tweezers to avoid damage to the right ventricular surface when the position of the purse-string suture is determined. When the tweezers was pointed toward the orifice of the VSD or the direction of blood flow, the position can be fine-tuned to ensure that the puncture site and PmVSD had the best entry angle and lay the foundation for the wire to smoothly pass through the VSD. The correct location of the incision and the purse-string suture can greatly increase the success rate of device closure and can reduce the operative time and surgical risk.

When multiple defects of AMS are very close together, it is easier to occlude the PmVSD completely through 
unfolded disk. Nevertheless, when the defects are divergent, PmVSD closure is a major challenge ${ }^{[14]}$. Devendran etc. ${ }^{[15]}$ considered that closure of multistream PmVSDs using a single device was not possible when the defects were far apart. But there were a few cases of success in our study. For example, we had one case with the two defects in the AMS far from each other. One defect was near the tricuspid septal leaflet, the other was close to the interventricular septum. The entry of guide wire into the VSD was extremely difficult. The guidewire and sheath could not enter the defect near the tricuspid septal leaflet. And only the defect close to the interventricular septum could be selected. If the guide wire is completely aligned with the direction of blood flow, entering the AMS is not difficult, but turning the guidewire 90 degrees to enter the left ventricle is very difficult. In this case, the purse-string can be positioned at a certain angle to reduce the difficulty for the guidewire to enter the left ventricle after it enters the AMS.

The reasons such as the defects of the multistream PmVSD being divergent, retrosternal PmVSD forming an Angle with the incision, led to that the procedure durations of PmVSDs closure via an ultra-minimal trans intercostal incision were longer than thats of DCVSDs closure ${ }^{[12]}$. So, the cooperation of the team and the cumulation of experience is more important. All members of the team should remain aware of the patient's condition and know how to deal with the situation changed during surgery. The surgeons and the echocardiologist should work closely to make the operation smoothly. Skillful surgical technique and proficient TEE guidance, wicth can reduce the duration of surgery, are very important for a successful procedure.

For AMS with a single stream, the left disk of the occluder can be all or partly pulled into the aneurysm to close PmVSD easily. However, the basilar part of the multistream PmVSD need be occluded through the left disk of the occluder be anchored on the left ventricle side of PmVSD. The surgical experience is summarized as follows. First, it is important to assess carefully PmVSD through TEE before select the appropriate occluders. Second, this challenge requires the surgeons and echocardiologist to have great patience and tacit cooperation. Third, when the guidewire enters the AMS, the surgeon can gently pull on the heart to reduce the angle and make it easier to allow the guidewire to enter the left ventricle. Fourth, a mild residual shunt ([?] $2 \mathrm{~mm})^{[16]}$ and low flowing velocity do not significantly impact the patient and can heal by itself.

Scholars had reported that the estimated incidence of arrhythmia, especially complete atrioventricular block, was $5.7-22 \%$ after transcatheter device closure ${ }^{[17,18]}$. The use of an oversized occluder is one of the main reasons for atrioventricular conduction block and other arrhythmias. Therefore, the size of occluders should be chosen as small as possible on the premise of success closure in order to avoid arrhythmia. In this study, the size of the device is approximately 1 (0.5-1.5) mm larger than the PmVSD diameter, and no obvious arrhythmia or complete atrioventricular block occurred.

The complications such as pericardial effusion, pleural effusion and hyperpyrexia contributed to increased postoperative hospital stay. These patients were not discharged until they had normal temperature and pericardial and pleural effusion improved.

Among them, 2 patients transferred to ventricular septal defect repair operation under direct visualization with a cardiopulmonary bypass. One patient was overweight, so the surgeon must pull the heart leftwards more than $3 \mathrm{~cm}$ to locate the precise position of the purse-string suture. Ventricular fibrillation occured when guide wire passed the PmVSD, which may be associated with excessive traction. The other patient had so large PmVSD that the device dislocated. The trans intercostal ultra-minimal incision is difficult to set up cardiopulmonary bypass quickly. PmVSD repair under visualization needs a new mid-sternum incision. After surgical repair, the presence of both a long and a short incision may affect the patient's appearance. We suggested that large PmVSD or obese children's PmVSD should be occluded via the minimally invasive inferior sternotomy approach. If closure fails, the incision can be extended.

\section{limitations}

There are several limitations of this study. It is a single center study. The age range of the children in this study was 10 months to 127 months; patients in other age groups are not included. A shorter-term follow-up was performed for 18-24 months. Our team will continue to expand the sample size and extend the follow-up 
time to provide more reliable and relevant information on this surgical method.

\section{Conclusions}

In conclusion, PmVSD closure via an ultra-minimal trans intercostal incision under TEE guidance is effective, feasible, safe and simple approach with less trauma. Surgical complications are rare. Therefore, promoting the application of this procedure in restrictive PmVSD is worthwhile.

\section{Conflict of interest}

We declare that we have no conflicts of interest.

\section{References}

1. Carminati M, Butera G, Chessa M, De Giovanni J, Fisher G, Gewillig M, Peuster M, Piechaud JF, Santoro G, Sievert H, Spadoni I, Walsh K; Investigators of the European VSD Registry. Transcatheter closure of congenital ventricular septal defects: results of the European Registry. Eur Heart J 2007;28:2361-8.

2. Xu XD, Liu SX, Bai Y, Zhang M, Zhao XX, Qin YW. Decreased tricuspid regurgitation following percutaneous closure of congenital perimembranous ventricular septal defect: immediate and 6-month echocardiographic assessment. Heart Vessels 2015;30:611-7.

3. Ei Sayed MH, Roushdy AM, Ei Farghaly H, Ei Sherbini A. Radiation Exposure in Children During the Current Era of Pediatric Cardiac Intervention. Pediatr Cardiol 2012;33: 27-35.

4. Wagdi P, Ritter M. Patient radiation dose during percutaneous interventional closure of interatrial communications. J Cardiol 2009;53:368-73.

5. Wang S, Zhuang Z, Zhang H, Zhen J, Lu Y, Liu J, Xu Z. Perventricular Closure of Perimembranous Ventricular Septal Defects Using the Concentric Occluder Device. Pediatric Cardiol 2014;35:580-6.

6. Chen ZY, Lin BR, Chen WH, Chen Q, Guo XF, Chen LL, Ge JB. Percutaneous device occlusion and minimally invasive surgical repair for perimembranous ventricular septal defect. Ann Thorac Surg 2014;97:1400-6.

7. Gan C, Lin K, An Q, Tang H, Song H, Lui RC, Tao K, Zhuang Z, Shi Y. Gan CP, Lin K, An Q, Shi YK. Perventricular device closure of muscular ventricular septal defects on beating hearts: Initial experience in eight children. J Thorac Cardiovasc Surg 2009;137:929-33.

8. Chen Q, Hong ZN, Zhang GC, Chen LW, Zhang QL, Lin ZW, Cao H. Intraoperative Device Closure of Isolated Ventricular Septal Defects: Experience on 1,090 Cases. Ann Thorac Surg 2018;105: 1797-1802.

9. Zhu D, Lin K, Tang ML, Feng Y, Ai Q. Midterm results of hybrid perventricular closure of doubly committed subarterial ventricular septal defects in pediatric patients. J Card Surg 2014;29:546-53.

10. Diab KA, Cao QL, Mora BN, Hijazi ZM. Device closure of muscular ventricular septal defects in children less than one year of age using the Amplatzer devices: Feasibility and outcome. Catheter Cardio Inte 2010;70:91-8.

11. Li HX, Zhang N, Guo WB, Zhang WL, Wang ZJ, Liang F, Zou CW. Peratrial device closure of perimembranous ventricular septal defects through a right parasternal approach. Ann Thorac Surg 2014;98:668-74.

12. Yu J, Ma LL, Ye JJ, Zhang ZW, Li JH, Yu JG, Jiang GP. Doubly committed ventricular septal defect closure using eccentric occluder via ultra-minimal incision. Eur J Cardiothorac Surg 2017;52:805-9.

13. Zhang GC, Chen Q, Cao H, Chen LW, Yang LP, Chen DZ. Minimally invasive perventricular device closure of ventricular septal defect in infants under transthoracic echocardiograhic guidance: feasibility and comparison with transesophageal echocardiography. Cardiovasc Ultrasound 2013;11:8-29.

14. Dilawar M, Numan M, El-Sisi, A. Gendi S M, Ahmad Z. Percutaneous Closure of Ventricular Septal Defect Associated with Tunnel-Shaped Aneurysm Using the Amplatzer Duct Occluder. Pediatr Cardiol 2008;29:366-70.

15. Devendran V, Koneti NR,Jesudian V. Transcatheter Closure of Multiple Perimembranous Ventricular Septal Defects With Septal Aneurysm Using Two Overlapping Amplatzer Duct Occluders II. Pediatr Cardio 2013;34:1963-5. 
16. Boutin C, Musewe NN, Smallhorn JF, Dyck JD, Kobayashi T, Benson LN. Echocardiographic follow-up of atrial septal defect after catheter closure by double umbrella device. Circulation 1993;88: 621-7.

17. Butera G, Carminati M, Chessa M, Piazza L, Micheletti A, Negura DG, Abella R, Giamberti A, Frigiola A. Transcatheter closure of perimembranous ventricular septal defects: early and long-term results. J Am Coll Cardiol 2007;18;50:1189-95.

Predescu D, Chaturvedi RR, Friedberg MK, Benson LN, Ozawa A, Lee KJ. Complete heart block associated with device closure of perimembranousventricular septal defects. J Thorac Cardiovasc Surg 2008;136:1223-8.

\section{Take-home figure}

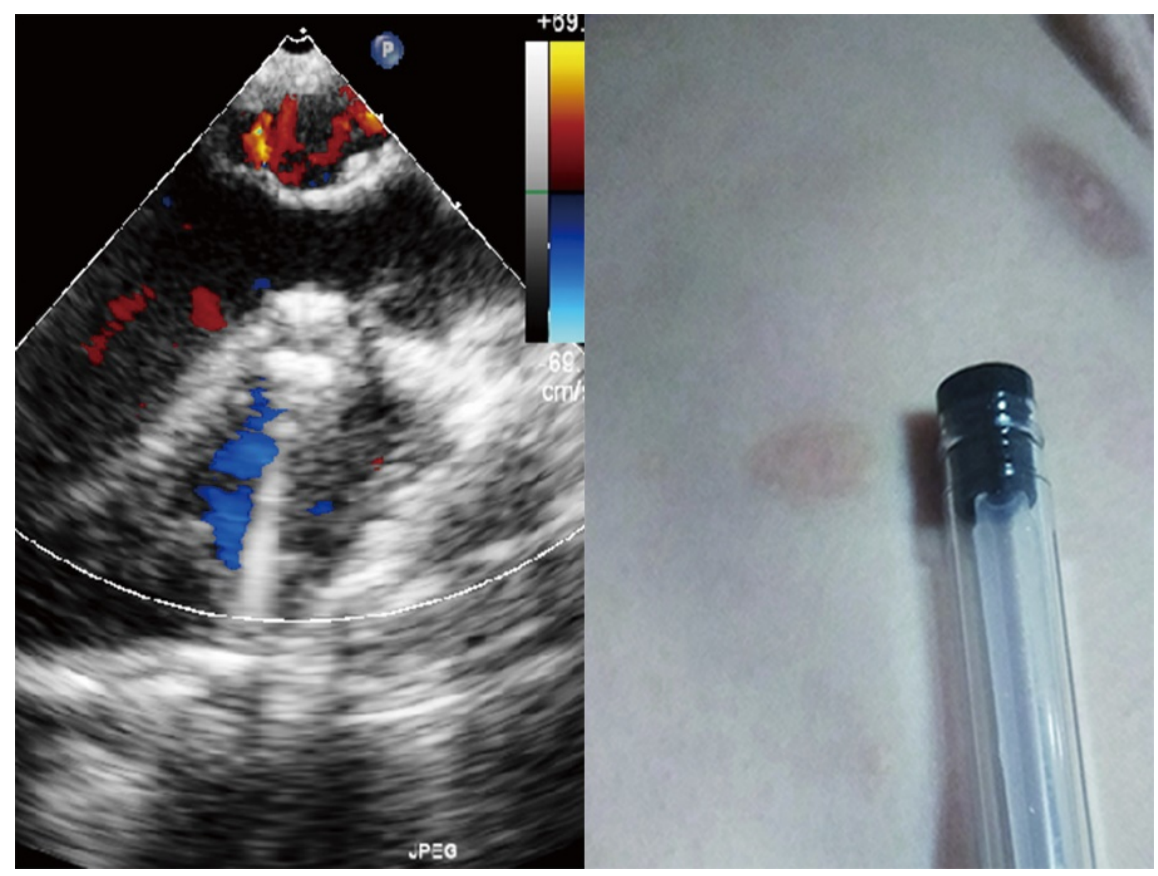

\section{One-Sentence Summary}

PmVSD was occluded using a concentric occluder device via a left parasternal ultra-minimal trans intercostal incision([?]1 cm) under TEE guidance in children, and the length of surgical scar was similar to the diameter of gel ink pen at one year.

\section{Figure 1}




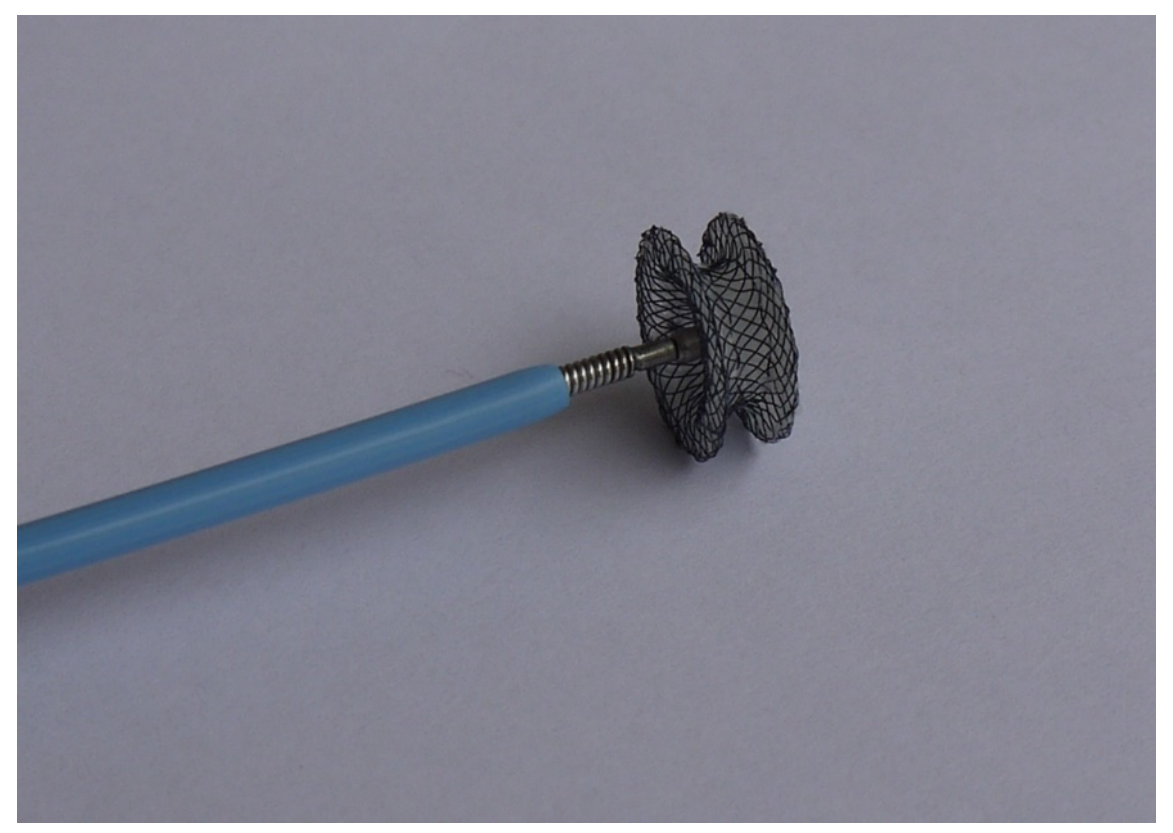

The concentric occluder was screwed onto the delivery cable, which traveled to the short loading sheath. Both disks of the concentric occluder have a diameter of $2 \mathrm{~mm}$ larger than that of the waist.

\section{Figure2}

A

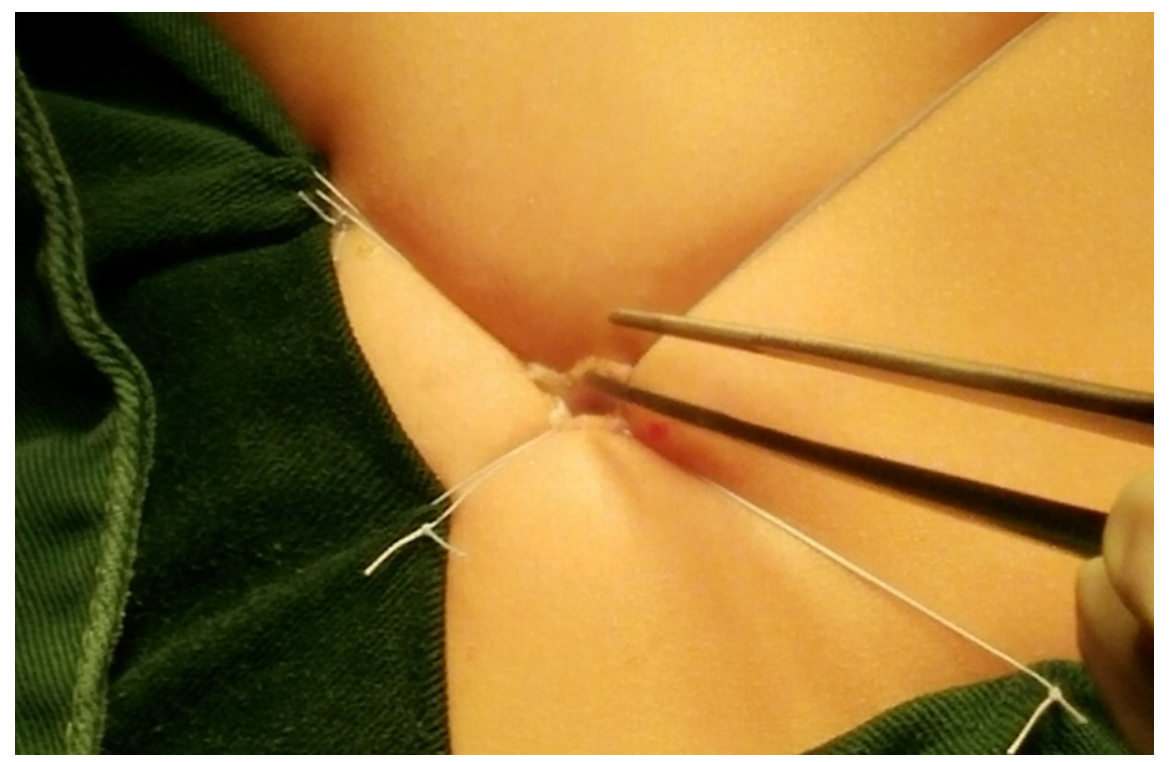




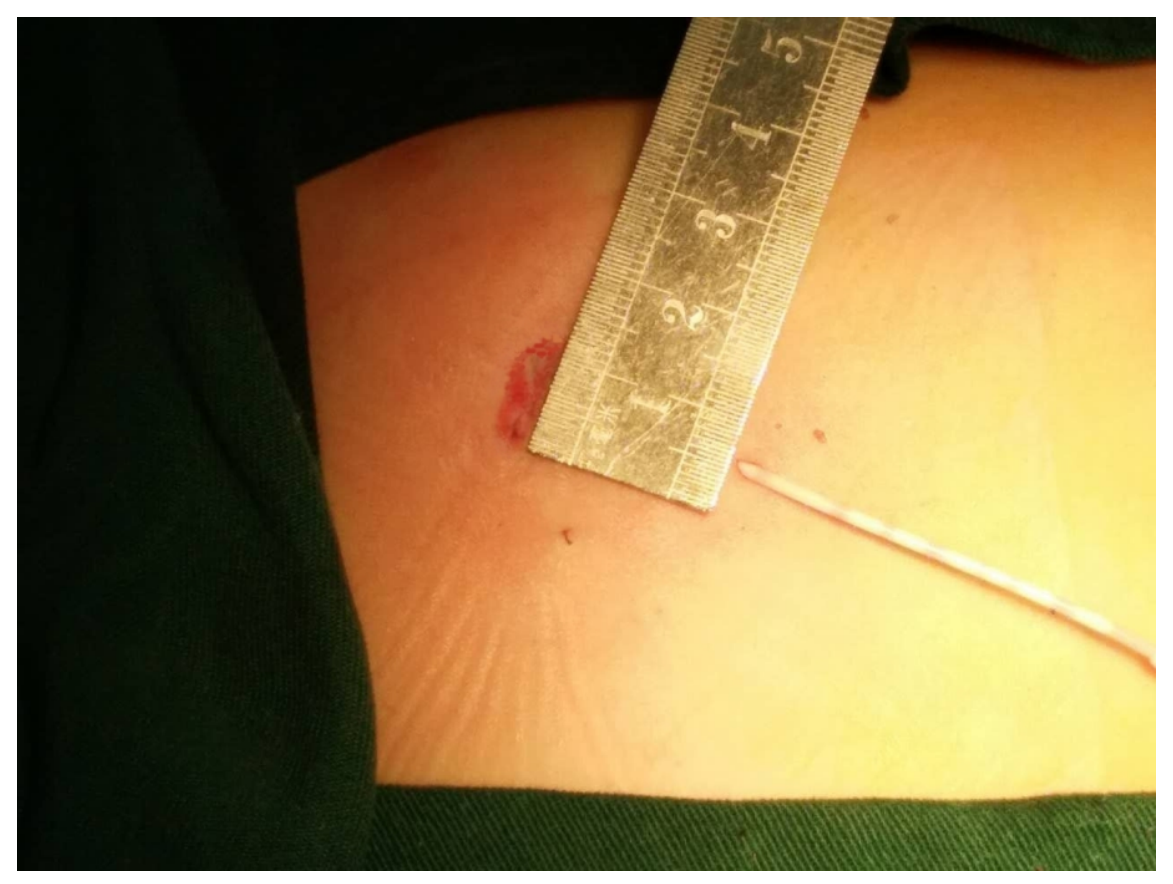

C

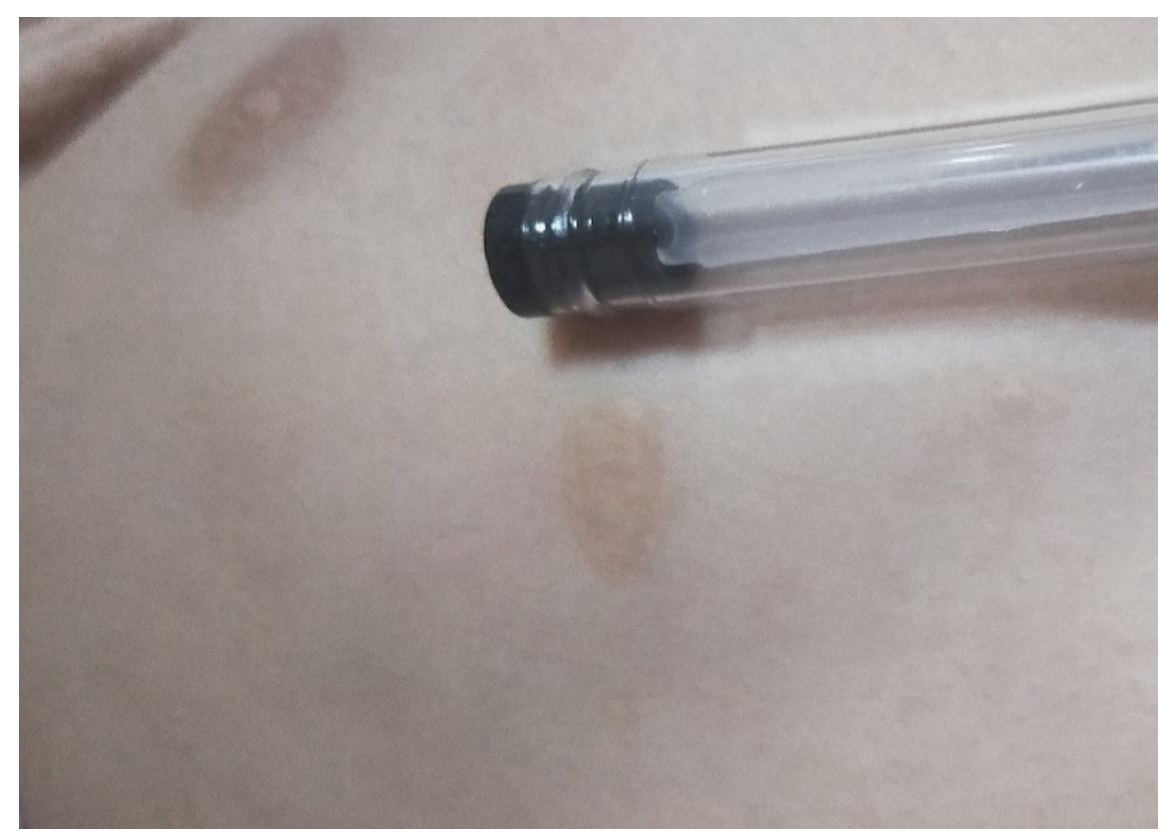

A: Hanging the pericardium with four sutures to expose the heart.

B: A $1.0 \mathrm{~cm}$ ultra-minimal intercostal incision used in children.

C: The length of surgical scar was compared with the diameter of gel ink pen at one year.

Figure 3 
A

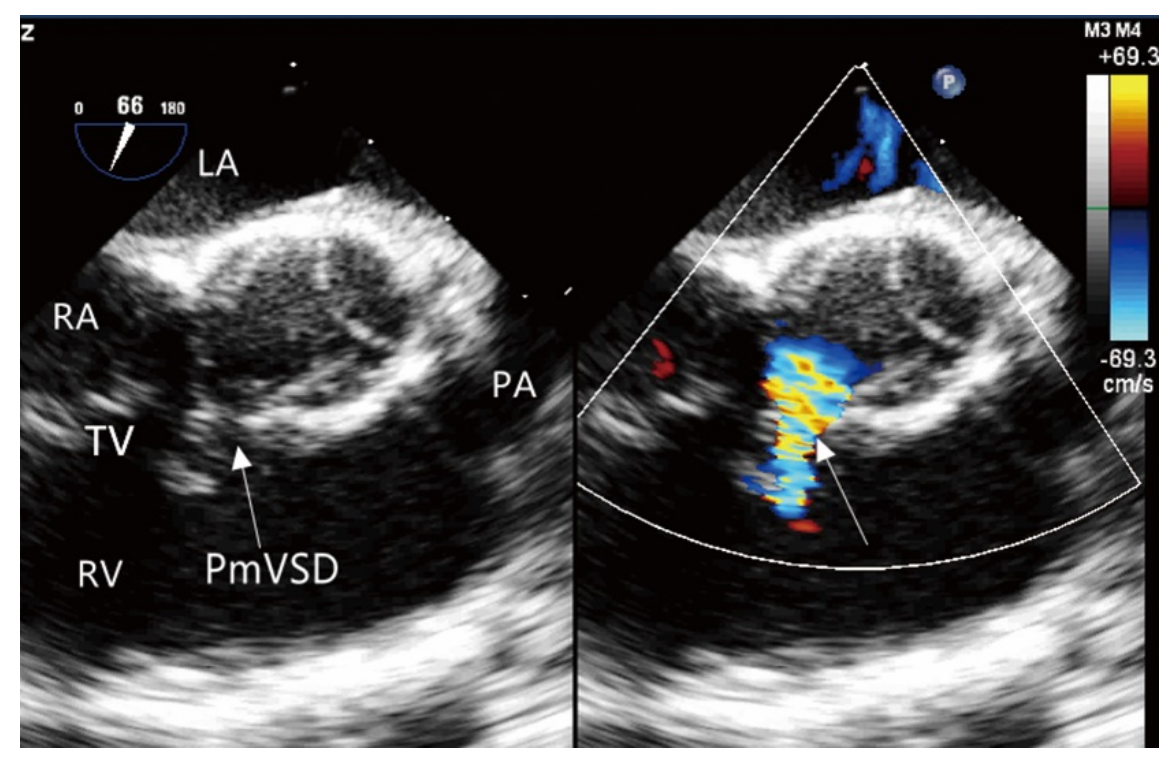

B

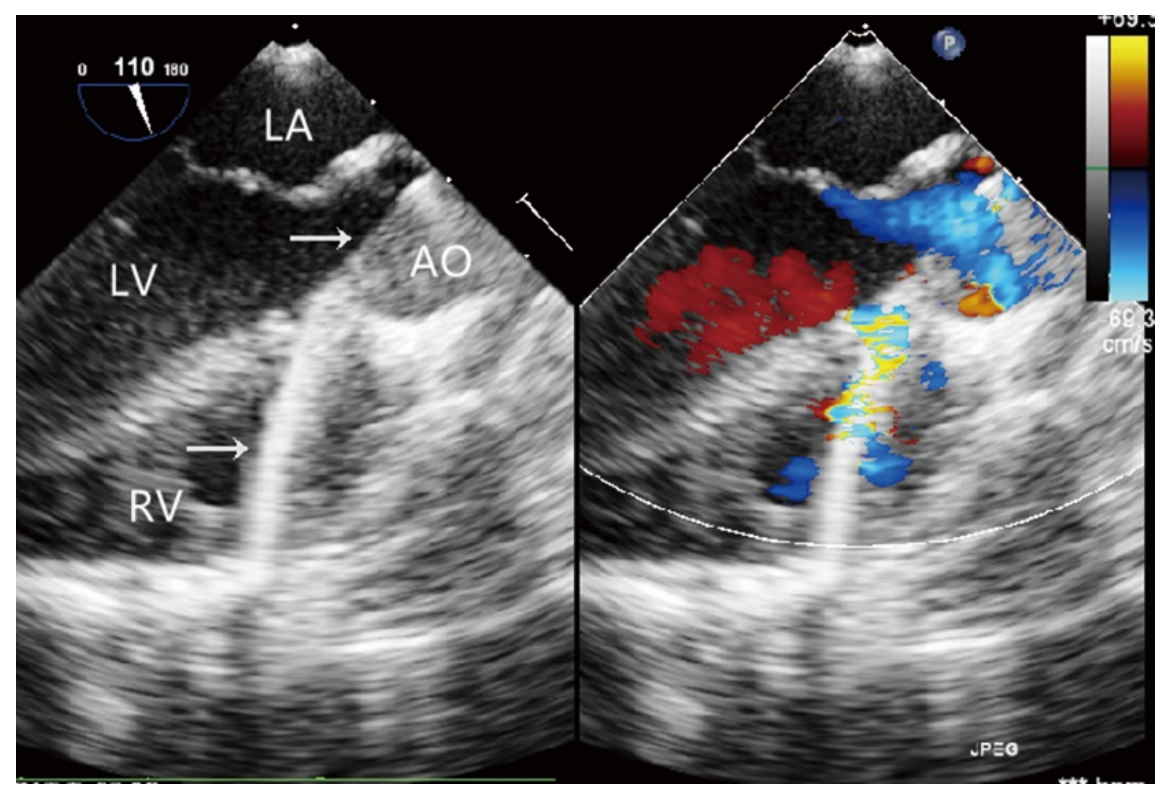

C 


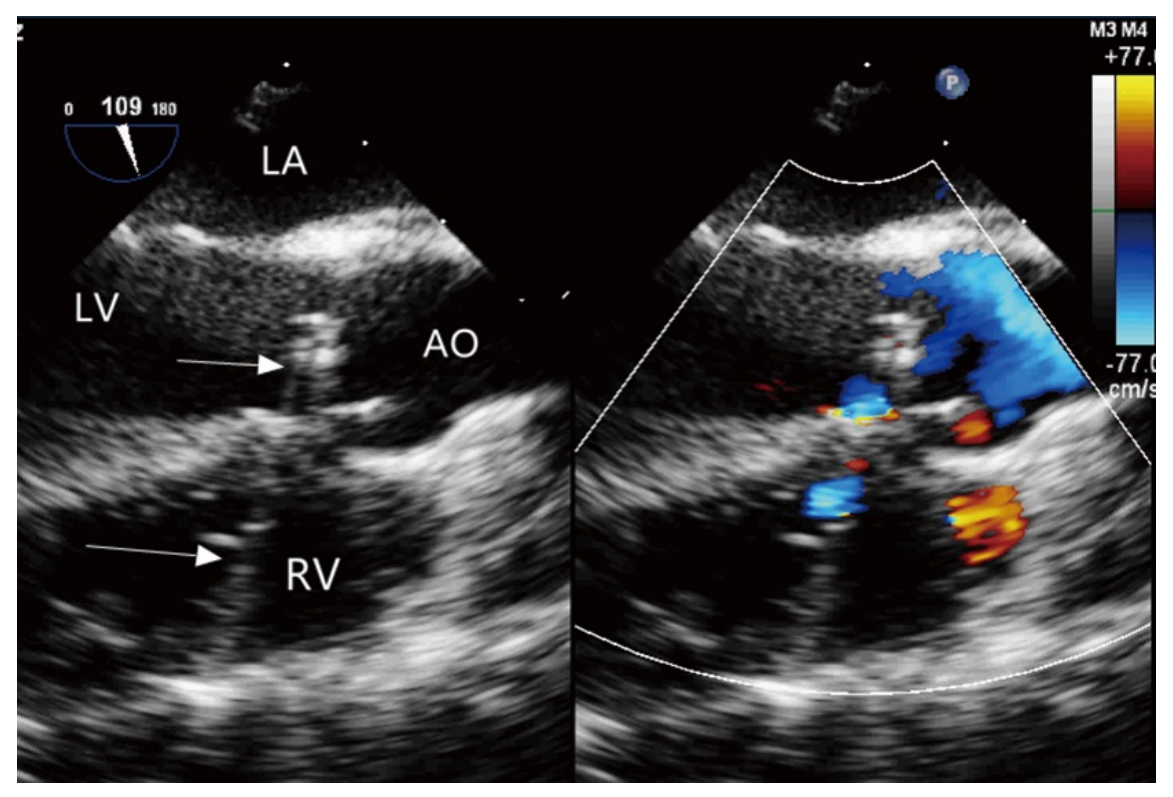

D

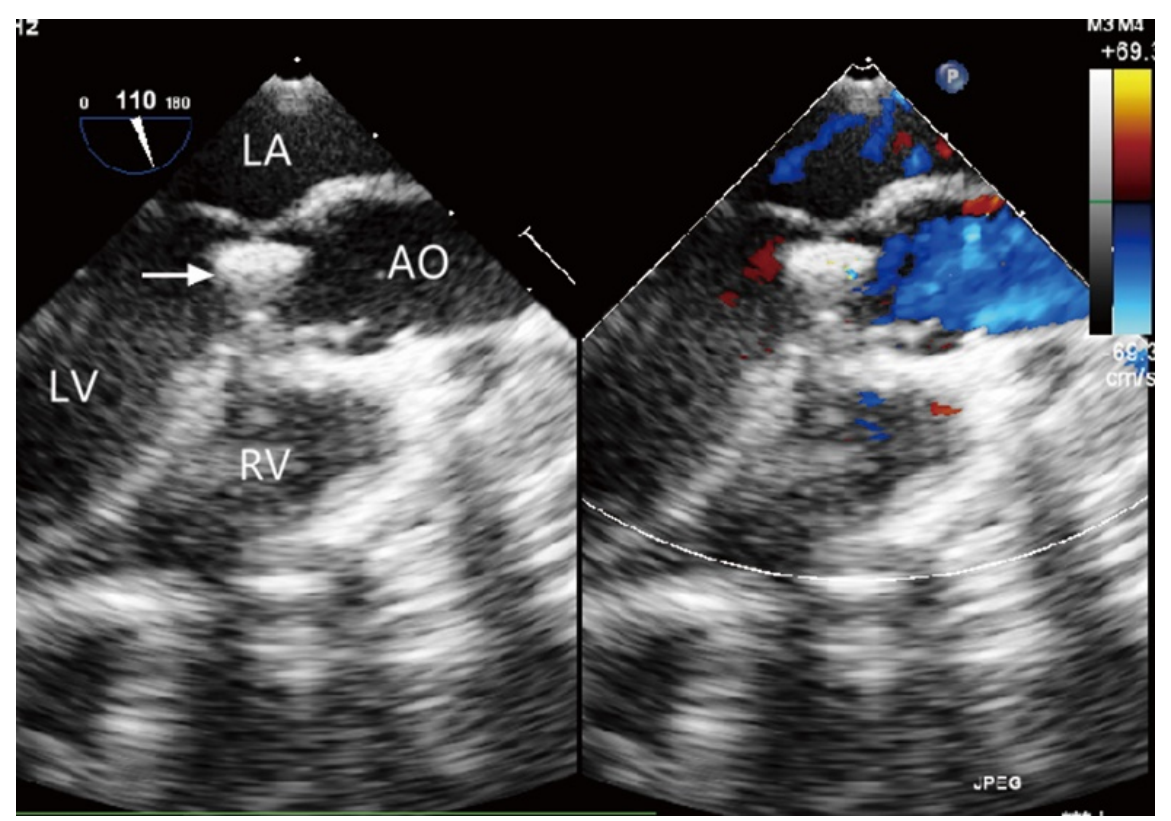

E 


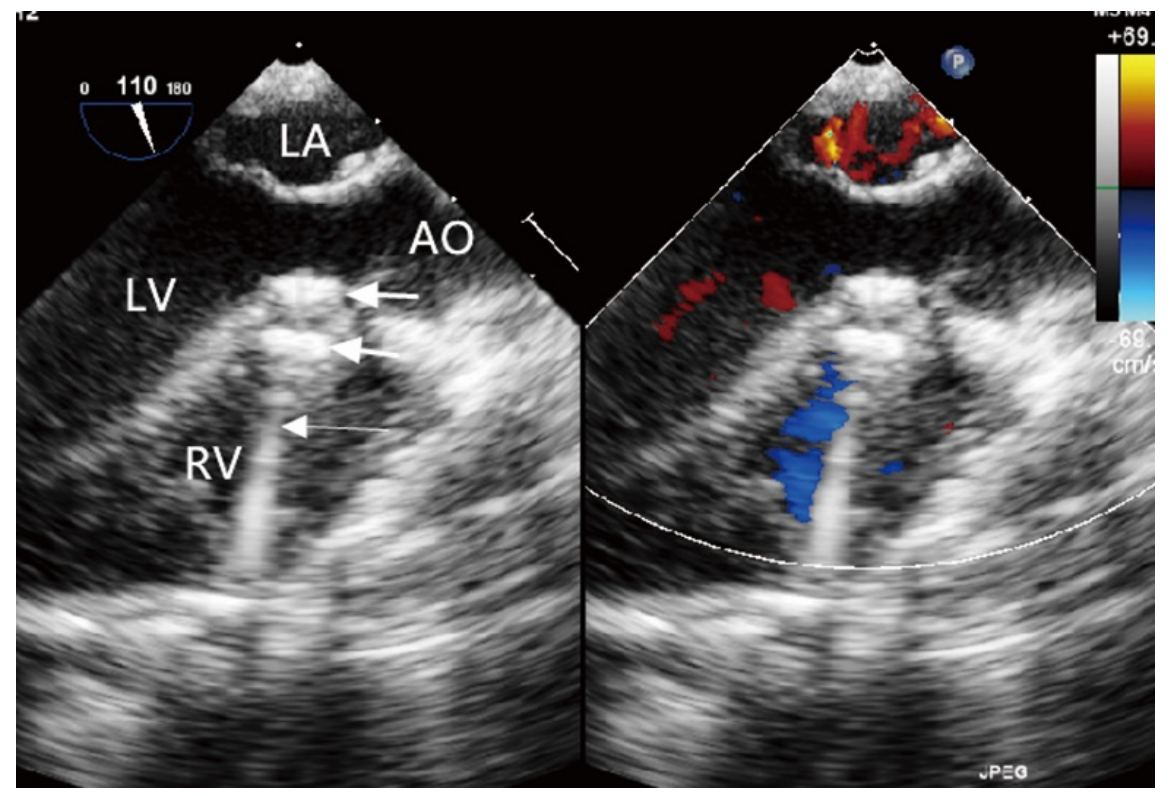

Procedures of PmVSD closure via ultra-minimal intercostal incision under the TEE guidance

A: A PmVSD (arrow) was found in the large artery short axial view on TEE.

B: The guidewire (arrow) was sent to the left ventricle through the PmVSD.

C: The delivery sheath and the dilator were introduced to the left ventricle over the guidewire. While the top of the sheath was confirmed to be in the left ventricle, the guidewire and the dilator were withdrawn and only the delivery sheath (arrow) was keep.

D: The short loading sheath loaded with the device was then connected to the long delivery sheath. The device was pushed to released the left disk (arrow) from the sheath.

E: The concentric occluder was placed in a proper position without residual shunt and influence on valve.

PmVSD: Perimembranous ventricular septal defect; TV: Tricuspid valve; LA: Left atrium;

LV: left ventricle;RV: Right atrium;AO: Aorta 

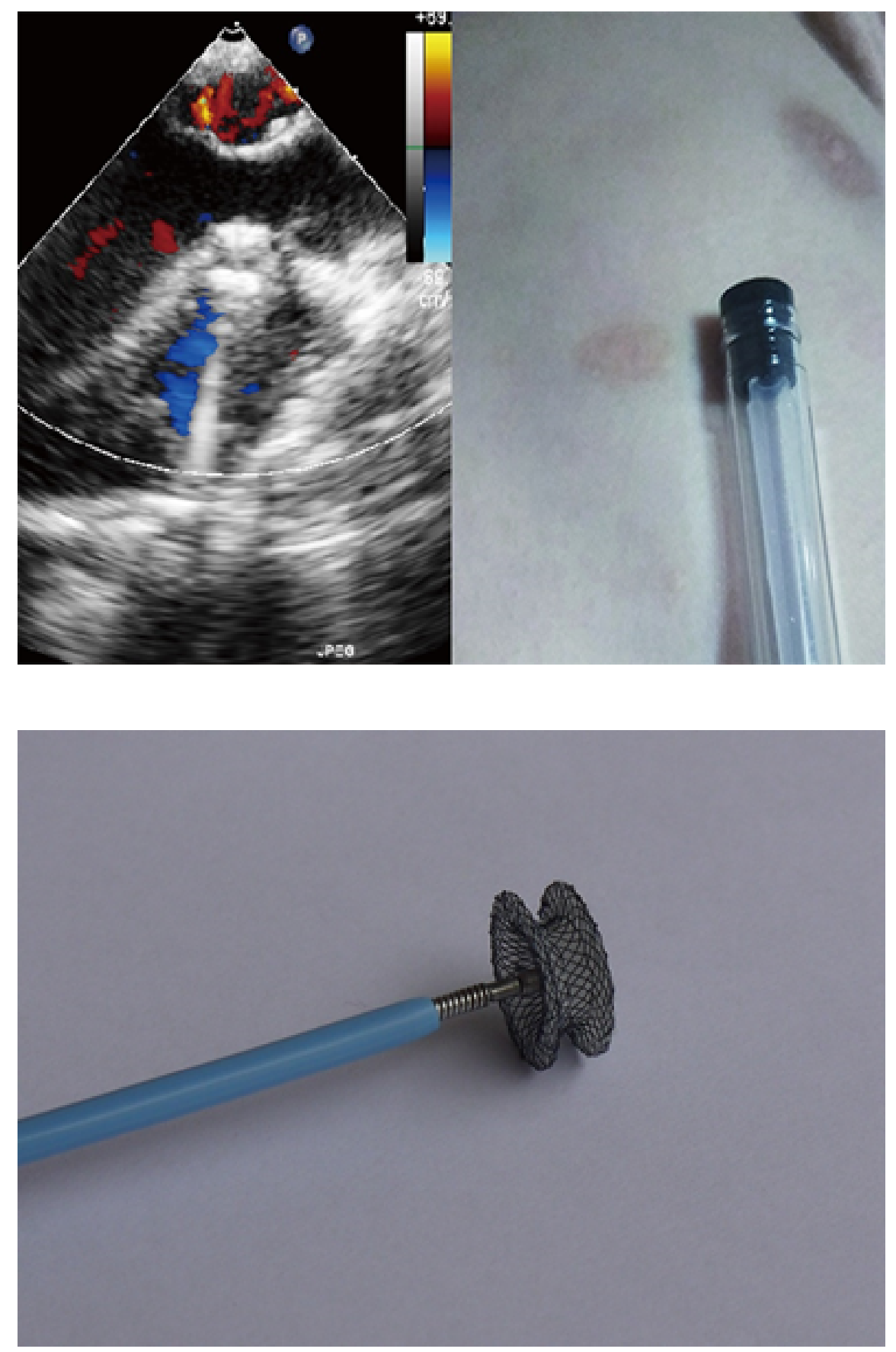

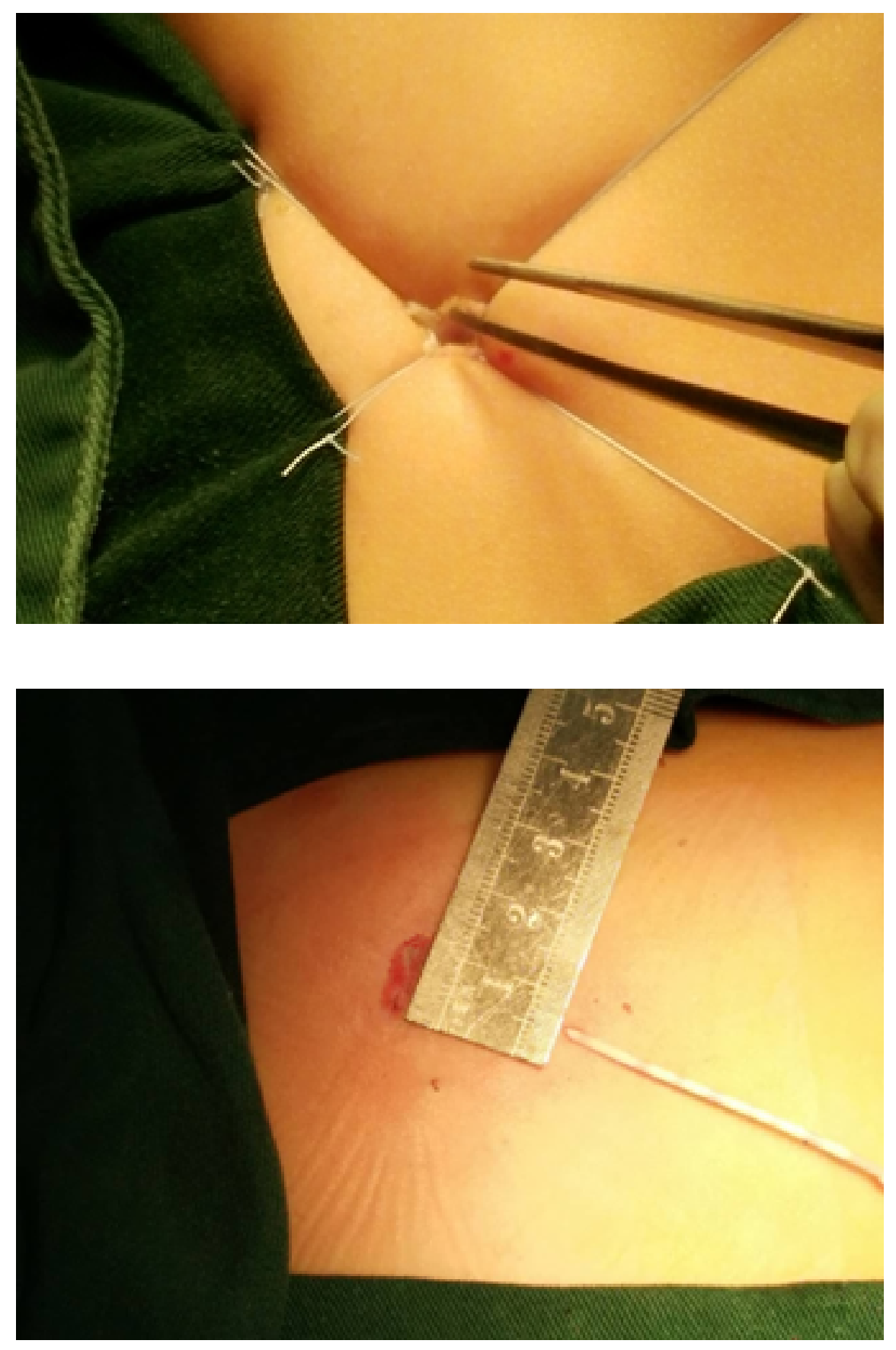


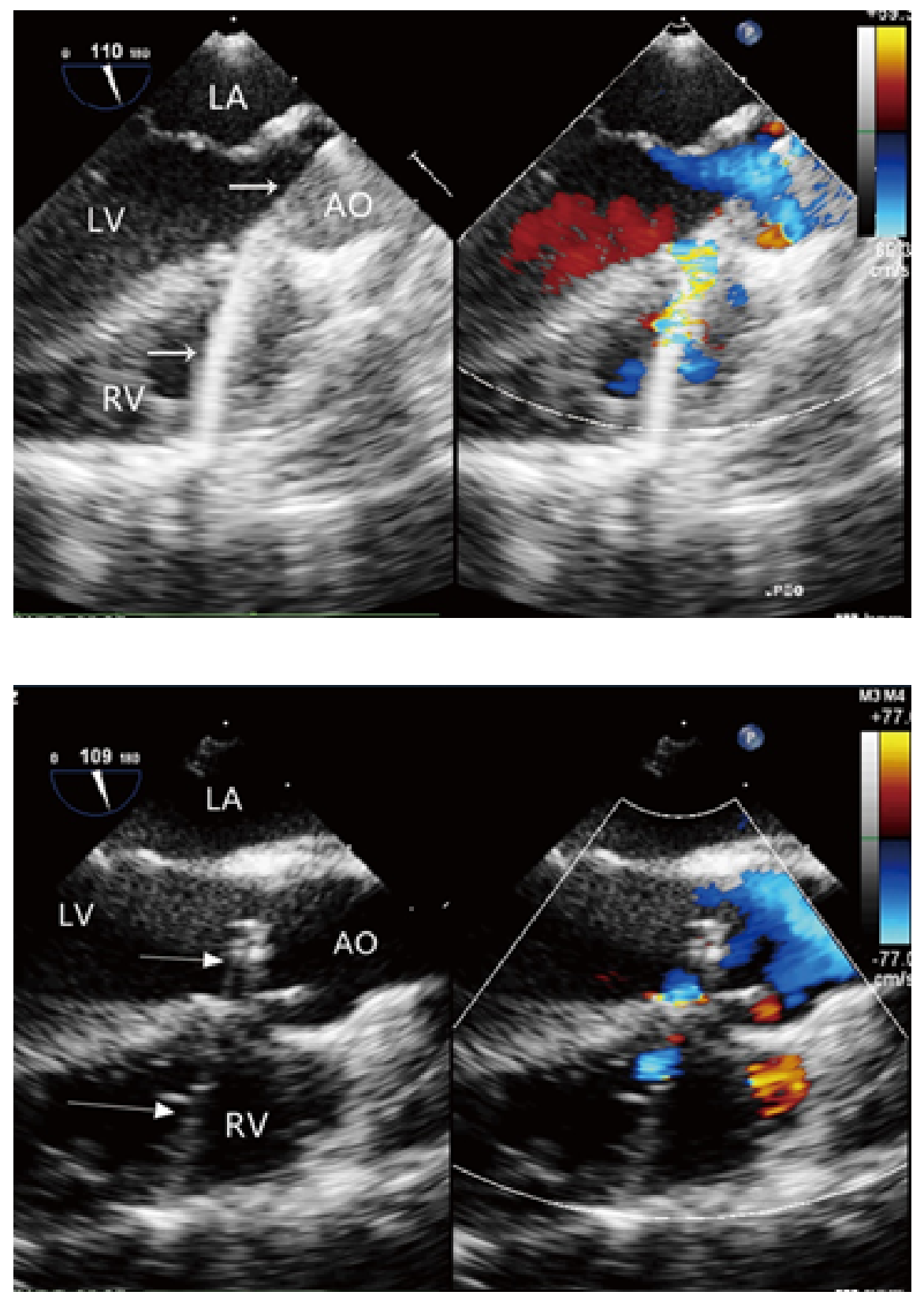

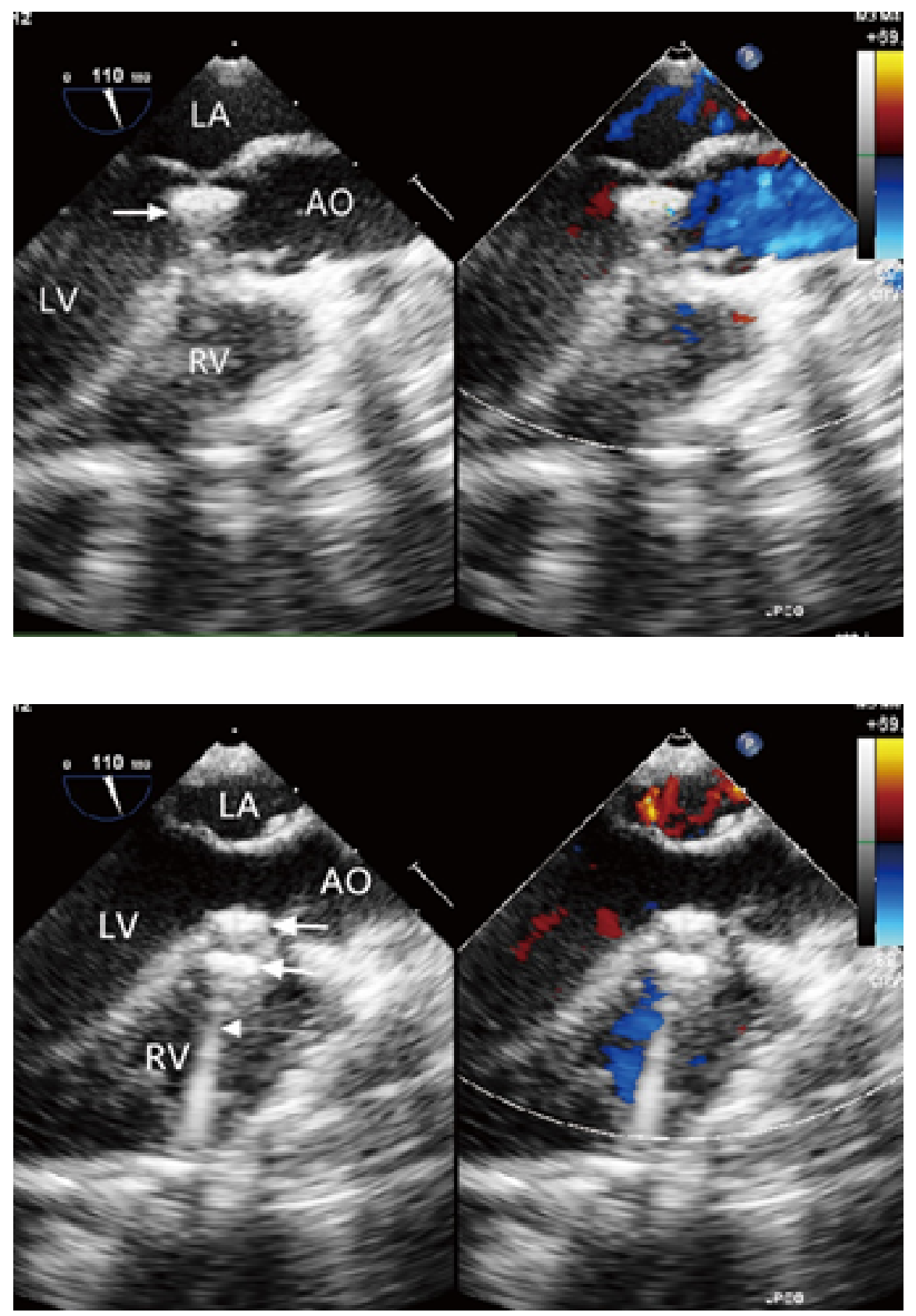